\title{
Iterative Multi-Frame Super-Resolution Image Reconstruction via Variance-Based Fidelity to the Data
}

\author{
Antigoni Panagiotopoulou \\ Chemical Engineering Department,University of Patras and Institute of Chemical Engineering Sciences of the \\ Foundation for Research and Technology-Hellas, Patras, Greece \\ anpanag@chemeng.upatras.gr
}

\begin{abstract}
Multi-frame Super-Resolution (SR) image reconstruction creates a single High-Resolution (HR) image from a sequence of Low-Resolution (LR) frames. Apart from resolution increment, blurring and noise removal is also achieved. In stochastic regularized methods, the SR problem is formulated by means of two terms, the data-fidelity term and the regularization term. In the present work, a novel estimator named Var- norm has been proposed for utilization in the data-fidelity term. This estimator presents a simple mathematical form based on the variance of the SR estimation error, i.e. on the difference between the estimated LR frame and the corresponding measured LR frame. The introduced Var-norm estimator is combined with the Bilateral Total Variation (BTV) regularization to formulate a novel SR method. The SR performance of the proposed method is directly compared with that of two SR techniques existing in the literature. Experimentation proves that the proposed method outperforms the existing methods.
\end{abstract}

\section{Keywords}

Super-resolution; Data-fidelity; Variance of Estimation Error; Influence Function; BTV Regularizer

\section{Introduction}

In multi-frame Super-Resolution (SR) image reconstruction, a High-Resolution (HR) image is reconstructed from a sequence of subpixel shifted, aliased Low-Resolution (LR) frames. The information associated to the relative displacement between the LR images has been investigated, and spatial resolution was increased by integrating these images into a single image. In fact, the image resolution enhancement achieved by the SR reconstruction techniques (Panagiotopoulou and Anastassopoulos, 2007, Katsaggelos et al., 2007) is far better than that accomplished by means of the interpolation techniques (Tsagaris et al., 2004, Panagiotopoulou and Anastassopoulos, 2008a). Nevertheless, there are limiting factors in the SR reconstruction techniques (Baker and Kanade, 2002, Pham et al., 2005). Thus, the resolution enhancement factor appears to be limited by the amount of aliasing and noise level. Both optical and sensor blurring are unavoidable.

The stochastic regularized SR image reconstruction problem (Park et al., 2003, Panagiotopoulou and Anastassopoulos, 2012a) has been vastly treated in the literature (Farsiu et al., 2004a, Pickup et al., 2004, Patanavijit and Jitapunkul, 2006a-d, Patanavijit et al. 2007a, Patanavijit and Jitapunkul, 2007b, Panagiotopoulou and Anastassopoulos, 2008b, Panagiotopoulou and Anastassopoulos, 2009, Pham et al., 2008, El-Yamany et al., 2007, El-Yamany et al., 2008a-b, Ivanonski et al., 2006, Panagiotopoulou, 2012). Various error norms or $\rho$-functions (Tukey, 1983, Black et al., 1998) have been utilized for the datafidelity term. Considering the SR image reconstruction as an iterative procedure, in each iteration an error norm is employed, as many times as the LR frames are, to measure the difference of each estimated frame from the corresponding measured frame. In relation to the treatment of the measurement, that is the frame difference, the employed $\rho$-functions can form different groups. The error norms that present unified treatment of the measurements can form one group and present functions of sole form, in contrast with functions of multiple forms depending on certain conditions being satisfied. The $L_{2}$ and $L_{1}$ error norms belong to the sole form group of unified measurements treatment (Farsiu et al. 2004a, Pickup et al., 2004, Patanavijit and Jitapunkul, 2006a-c). Practically, the influence functions (Tukey, 1983, Black et al., 1998) of the particular estimators treat all measurements equally regarding the characterization of their bias introduced in the solution. However, certain error norms do not present unified treatment of the measurements and distinguish between usable 
and not usable measurements. The latter is regarded outliers and treated specially (Farsiu et al. 2004a). These error norms that can formulate another group, present an outliers rejection threshold. The influence functions of the particular estimators characterize differently the bias introduced in the solution by each measurement in dependence on the considered usability of the measurement. The Huber, Lorentzian, Tukey and Gaussian-weighted $L_{2}$ error norms belong to the above-mentioned group (Patanavijit and Jitapunkul, 2006d, Patanavijit et al. 2007a, Patanavijit and Jitapunkul, 2007b, Panagiotopoulou and Anastassopoulos, 2008b, Panagiotopoulou and Anastassopoulos, 2009, Pham et al., 2008). Moreover, adaptive M-estimation SR approaches have been presented with tunable threshold of outliers rejection (El-Yamany et al., 2007, El-Yamany et al., 2008a-b). Additionally, a selective approach to SR re-construction has been presented in (Ivanovski et al., 2006).

The present work introduces a stochastic regularized SR image reconstruction technique with a novel estimator employed in the data-fidelity term of the SR problem formulation. The introduced estimator named Varnorm is based on the variance of the SR estimation error. In fact, the influence function of this estimator changes in relation to the variance of the difference between the estimated frame and the corresponding measured frame. Thus, the values of the weights which are given to the arguments of the influence function change temporarily. Specifically, the arguments of the influence function are given large or small weights in case of correct or incorrect HR estimate, respectively. Thereafter, the characterization of the bias introduced in the solution by each measurement is automatically renewed during the SR reconstruction procedure and the proposed estimator provides for only the correct measurements strongly affecting the solution. In the introduced SR technique the Bilateral TV (BTV) regularization (Panagiotopoulou and Anastassopoulos, 2009) was employed for the regularization term. Experimentation with real and synthesized sequences of frames was carried out. The experimental results prove that the proposed SR method predominates over the SR techniques presented in (Farsiu et al., 2004a, Patanavijit and Jitapunkul, 2006b) that utilize the $L_{1}$ and $L_{2}$ estimators, respectively, in combination with the BTV regularization.

The present paper considers stochastic regularized SR image reconstruction from the data-fidelity point of view. Thus, in the above-mentioned literature review the different error norms are categorized into groups by taking into account how they treat measurements, during the iterative SR reconstruction procedure, through their employment in the data-fidelity term. According to the above-mentioned grouping criterion, the novel estimator which was proposed in the present work can be assigned in the same group with the $L_{2}$ and $L_{1}$ error norms, which is the sole form group of unified measurements treatment. So, in this work experimentation was carried out to directly compare the proposed error norm only with the $L_{2}$ and $L_{1}$ error norms since all the other estimators reviewed from the literature handle measurements differently compared to these three estimators. The remainder of the paper is organized as follows. In the second section the formulation and solution of the SR image reconstruction problem are discussed. The implementation of the SR reconstruction procedure is also described. The issue of fidelity to the measured data is discussed in the third section. Afterwards, the conducted experiments are presented and the conclusions are drawn.

\section{The Problem of Super-Resolution Image Re- construction}

\section{Formulation and Solution}

SR image reconstruction is an inverse problem. Specifically, the source of information that is the HR image, is estimated from the observed LR images. In the context of stochastic regularized SR reconstruction techniques, the formulation of the SR problem takes place by means of two terms, the data-fidelity term and the regularization term (Park et al., 2003, Panagiotopoulou and Anastassopoulos, 2012a). Actually, SR reconstruction is a minimization problem since the SR estimate minimizes some cost function (Farsiu et al., 2004a-b). The solution HR image, denoted as $X$, can be expressed as follows:

$$
X=\underset{X}{\operatorname{ArgMin}}\left[\sum_{i=1}^{N} \rho\left(M_{i} X-Y_{i}\right)+\lambda r(X)\right]
$$

where $\sum_{i=1}^{N} \rho\left(M_{i} X-Y_{i}\right)$ stands for the data-fidelity term. The particular term consists of a cost function, or objective function, named $\rho$-function or error norm which measures the difference between the projected estimate of the HR image $M_{i} X$ and each LR frame $Y_{i}$, thus assuring a certain fidelity of the final solution to the measured data. In fact, $M_{i} X$ stands for the estimated LR frame and $Y_{i}$ is the corresponding measured LR frame. The operator $M$ denotes the imaging system which can be modeled as separate 
operators representing shifting, blurring and downsampling operations. The symbol $N$ denotes the LR frames number.

As far as $r(X)$ is concerned, it is the regularization term and consists of a cost function which poses a penalty on the estimated HR image with the intention to direct it to a better formed solution (Farsiu et al., 2004b, Engl et al., 2000). The regularization term compensates the missing measurement information with some general prior information about the desirable HR solution (Engl et al., 2000). In this work the BTV regularization (Panagiotopoulou and Anastassopoulos, 2009) was employed and thus incorporated as a-priori knowledge about the solution. This is a robust regularization technique which exhibits the capability of performing adaptive smoothing based on the local structure of the image. With regard to the coefficient $\lambda$, it is called regularization parameter and determines the strength by which regularization imposes constraints on the HR estimate.

By employing iterative solution method based on gradient, equation (1) is transformed into:

$$
\begin{aligned}
X_{n+1} & =X_{n}-\beta\left\{\left[\sum_{i=1}^{N} \rho\left(\mathrm{M}_{i} X-Y_{i}\right)\right]^{\prime}+\lambda r^{\prime}(x)\right\} \\
& =X_{n}-\beta\left\{\sum_{i=1}^{N} M_{i}^{T} \psi\left(M_{i} X_{n}-Y_{i}\right)+\lambda r^{\prime}\left(X_{n}\right)\right\}
\end{aligned}
$$

The function $\psi$ stands for the influence function (Black et al., 1998) of the $\rho$-function employed whilst $r^{\prime}$ is the derivative of $r$. The parameter $\beta$ is a scalar that determines the step size in the direction of the gradient. Regarding the parameter $n$, it denotes the number of iterations and takes integer values. $X_{n}$ and $X_{n+1}$ stand for the HR estimates which are calculated during iterations $n$ and $n+1$, respectively.

\section{Implementation}

The present work considers SR image reconstruction as an iterative procedure. MATLAB is used for the implementation. In the first iteration of the SR procedure, an initial HR estimate $X_{0}$ that could be created via interpolating a LR frame, serves for input. All the frames of the employed LR sequence also serve for inputs. In the following, the calculation of the HR estimate $X_{1}$ during iteration 1 is described, equation (2). Firstly, the initialization $X_{0}$ is shifted per the motion vector of frame 1, i.e. per the horizontal and vertical shifts that frame 1 exhibits. The shifted $X_{0}$ is convolved with the function which stands for the
Point Spread Function (PSF) of the camera having captured the LR sequence of frames. The obtained result is down-sampled and the outcome represents the estimated LR frame 1 . Then, the subtraction of the measured frame 1 from the estimated frame 1 takes place. The result is a matrix whose elements are then altered as mandated by the $\psi$ - function of the estimator utilized for the data-fidelity term. The newly obtained matrix firstly is up-sampled via zero filling and secondly is convolved with the transpose of the camera PSF. Finally, the outcome is shifted in opposite direction to that indicated by the motion vector of frame 1. The specific final outcome represents component 1 of the sum derivative of equation (2). By following the entire above-mentioned procedure as many times as the LR frames are, employing different frame each time, the rest components of the sum derivative are obtained. The summation of all these components gives the sum derivative. Afterwards, the first derivative of the regularizer employed was calculated. The initialization image $X_{0}$ serves as input for the particular calculation. With regard to the BTV regularizer, its mathematical form and its derivative can be found in (Panagiotopoulou and Anastassopoulos, 2009). By means of (2) with $n=0$ the SR estimate $X_{1}$ now can be calculated. In the second iteration, the SR estimate $X_{1}$ as well as all the LR frames serve for inputs, and the previously described procedure is followed to produce $X_{2}$. After $n$ iterations, the SR estimate $X_{n}$ results.

\section{Fidelity to the Measured Data}

\section{Introduction}

In the formulation of the SR image reconstruction problem (1), preservation of fidelity to the measured data is accomplished via the data-fidelity term. At the particular term, the LR frames resulting from shifting, blurring and downsampling the estimated HR image are compared with the corresponding measured LR frames, in order to assess the correctness of the HR estimate. The selection of the error norm utilized in the data-fidelity term is critical since a solution highly consistent with the measured data is desired. Actually, the behavior of the employed $\rho$-function can be analyzed by means of its influence function $\psi$ ((Tukey, 1983, Black et al., 1998). The latter is proportional to the derivative of $\rho$ and characterizes the bias that is introduced in the solution by a particular measurement. Thus, essentially the influence function controls the interaction of measurements and solution. The 
calculated difference between the projected estimate of the HR image and each LR frame stands for measurement.

In the literature (Farsiu et al., 2004a, Pickup et al., 2004, Patanavijit and Jitapunkul, 2006a-d, Patanavijit et al. 2007a, Patanavijit and Jitapunkul, 2007b, Panagiotopoulou and Anastassopoulos, 2008b, Panagiotopoulou and Anastassopoulos, 2009, Pham et al., 2008, El-Yamany et al., 2007, El-Yamany et al., 2008a-b, Ivanonski et al., 2006, Panagiotopoulou, 2012) various estimators have been employed for the data-fidelity term. As far as unified treatment of the measurements is concerned, the $L_{2}$ and $L_{1}$ error norms have been utilized (Farsiu et al. 2004a, Pickup et al., 2004, Patanavijit and Jitapunkul, 2006a-c). The particular error norms present functions of sole form, in contradiction with functions of multiple forms depending on certain conditions satisfied (Patanavijit and Jitapunkul, 2006d, Patanavijit et al. 2007a, Patanavijit and Jitapunkul, 2007b, Panagiotopoulou and Anastassopoulos, 2008b, Panagiotopoulou and Anastassopoulos, 2009, Pham et al., 2008). Thus, the quadratic error norm:

$$
\rho_{L_{2}}(x)=x^{2}
$$

has the linear $\psi$-function:

$$
\psi_{L_{2}}(x)=2 x
$$

that is plotted in Fig. 1(a). This influence function increases linearly and without bound. The quadratic error norm is very sensitive to outliers since it gives them too much influence. An increase in robustness and rejection of outliers can take place via an error norm which increases less rapidly than $x^{2}$. Such an estimator is the $L_{1}$-norm estimator:

$$
\rho_{L_{1}}(x)=|x|
$$

and converges to the median estimator, whose breakdown bound presents the largest possible value for a location estimator which treats observations on each side of the estimate symmetrically (Tukey, 1983). Its influence function is given by:

$$
\psi_{L_{1}}(x)=\operatorname{sign}(x)
$$

The $L_{1}$-norm gives all measurements a constant weight of one, as shown in Fig. 1(b).

Both above-mentioned estimators $L_{1}$ and $L_{2}$ present influence functions that treat all measurements equally regarding the characterization of their bias introduced in the solution. The estimator Var-norm proposed in this work presents unified treatment of the measurements thus exhibiting sole function form, like the $L_{1}$ and $L_{2}$ estimators. Nevertheless, the influence function of the Var-norm estimator does not treat all measurements equally with regard to the characterization of their bias introduced in the solution. Actually, the introduced estimator Var - norm exhibits the inherent property to determine what effect each measurement should have on the solution and presents an adaptive nature. Therefore, employing the proposed estimator during the SR image reconstruction procedure, the characterization of the bias introduced in the solution by each measurement is renewed.

\section{The Proposed Estimator}

The estimator utilized for the data-fidelity term measures the difference of each estimated LR frame from the corresponding measured LR frame in each iteration of the SR reconstruction procedure. Actually, the influence function of the employed error norm takes as arguments the elements of the matrix resulting from the subtraction of the estimated frame from the measured frame. With regard to the estimated frame, it can be obtained from the estimated SR reconstructed image via considering the acquisition process of the particular frame. Small difference of the LR frames under comparison means successful HR estimation. Therefore, the employed influence function should award the small differences between the compared frames and penalize large particular differences. Specifically, the influence function arguments should be given large or small weights in case of correct or incorrect HR estimate, respectively. In this way, only the correct measurements strongly affect the solution and the SR reconstruction procedure is not dominated by erroneous HR estimates. Then the utilized estimator provides for preserving fidelity to the measured LR frames.

The novel estimator proposed in the present work is named Var-norm and presents the following $\rho-$ and $\psi$ - functions

$$
\begin{aligned}
& \rho_{\text {var }}(x)=\frac{x^{2}}{R+2 \operatorname{var}(a b s(F))} \\
& \psi_{\text {var }}(x)=\frac{x}{R+\operatorname{var}(a b s(F))}
\end{aligned}
$$

$F$ denotes a matrix that is the difference between the estimated LR frame and the corresponding measured LR frame. Regarding $x$, it stands for element of the matrix $F$. The symbol $a b s$ represents absolute value and var stands for variance. The variance of the matrix $F$ is equal to the variance of the row vector which contains the variance of each column of $F$. $R$ is a parameter which accounts for stability of the estimator and its value is set in relation to the iterations of the SR reconstruction procedure. In fact, to assure stability the value of $R$ is renewed during each iteration. 


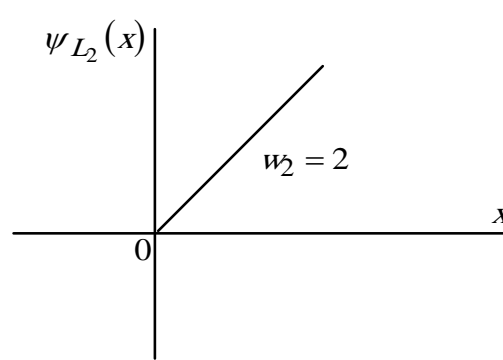

(a)

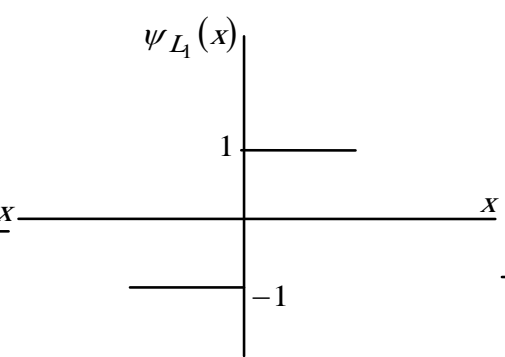

(b)

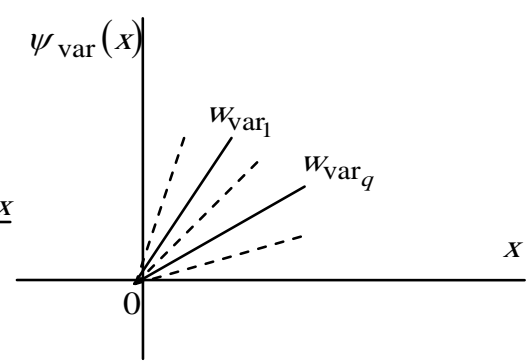

(c)

FIG. 1 THE $\psi$ - FUNCTIONS OF THE DIFFERENT ESTIMATORS. THE SYMBOL $w$ STANDS FOR SLOPE. (a) $L_{2} \psi-$ FUNCTION. (b) $L_{1}$ $\psi$ - FUNCTION. (c) THE PROPOSED Var - NORM $\psi$ - FUNCTION THAT PRESENTS VARIANT SLOPE, WHICH ALLOWS RENEW THE CHARACTERIZATION OF THE BIAS INTRODUCED IN THE SOLUTION BY EACH MEASUREMENT.

Actually, the elements of the matrix $F$ are estimation errors. In case the LR frame has not been correctly estimated, which denotes incorrect HR estimate, the elements of the matrix $F$ have large absolute values. Therefore, $\operatorname{var}(a b s(F))$ that is the variance of the estimation error also presents large value. In the specific case due to the proposed influence function (8), the values of the elements of $F$ will be decreased. The particular values denote incorrect estimation, so they will be penalized. On the contrary, in case the LR frame has been correctly estimated, denoting successful HR estimate, the introduced influence function increases the values of the elements $x$. Consequently, in each iteration of the SR re-construction procedure for each estimated LR frame the weight which is given to the argument of the influence function is automatically tuned, thus the influence function is altered. Fig. 2 presents pseudo-code that describes the utilization of the influence function $\psi_{\mathrm{var}}$ in the SR reconstruction procedure. Since $\operatorname{var}(a b s(F))$ is a single number, the SR estimation error is controlled globally for each LR image.

$$
\begin{aligned}
& \begin{array}{l}
F=\text { LRest }-L R m e a s ; \\
\text { //LRest denotes the estimated LR frame }
\end{array} \\
& \text { for }(i=1 ; i<s z 1+1 ; i++)\{/ / s z 1 \times s z 2 \text { is the size of matrix } F \\
& \text { for }(j=1 ; j<s z 2+1 ; j++)\{ \\
& \quad F[i][j]=F[i][j] /(\operatorname{var}(a b s(F))) ; / / F[i][j] \text { denotes } \\
& \text { //element of row i and column } j \\
& \text { \}end for } \\
& \text { \}end for }
\end{aligned}
$$

FIG. 2 PSEUDO-CODE DESCRIBING THE UTILIZATION OF THE PROPOSED INFLUENCE FUNCTION IN THE SR RECONSTRUCTION. THIS CODE PRESENTS THE MODIFICATION OF THE DIFFERENCE BETWEEN THE ESTIMATED AND THE MEASURED LR FRAMES PERFORMED BY THE PROPOSED ESTIMATOR, TO ASSURE CONSISTENCY OF THE SR ESTIMATE WITH THE MEASURED DATA.

Fig. 1 demonstrates graphical representation of the $\psi$-functions of the estimators under consideration.
Fig. 1(a) and (b) show the influence functions of the $L_{2}-$ and $L_{1}-$ norm estimators, respectively. Concerning the influence function of the proposed estimator Var-norm, it is plotted in Fig. 1(c). The particular function is a straight line. The point of intersection between the graph of the function and the $y$-axis is constant and equals zero. However, the line slope that equals $\frac{1}{R+\operatorname{var}(a b s(F))}$ varies according to the estimation error which is represented by $F$. Thus, estimation errors ranging from small to large values make the line of the influence function steep or swallow, respectively. In detail, a small estimation error gives a large value to the line slope. Thereafter, the related measurements strongly affect the solution. Contrarily, a large estimation error makes the value of the line slope small. So, the effect that the associated measurements have on the solution is weakened. In Fig. 1(c) the slope $w_{\mathrm{var}_{1}}$, that corresponds to small error, has greater value than the slope $w_{\mathrm{var}_{q}}$, that associates to large error. Consequently, the $\psi$-function of the proposed estimator Var - norm presents variant slope which allows renew the characterization of the bias introduced in the solution by each measurement. Contrarily, the $\psi$-functions of the $L_{2}-$ and $L_{1}-$ norm estimators do not present the capability of renewing the bias characterization. Thereafter, the proposed estimator outperforms the already existing estimators.

\section{Analysis from the Estimation Point of View}

The class of estimators that offer the greatest advantages in performance, flexibility and convenience are the $M$ - estimators. Through the choice of objective functions $M$ - estimation generalizes least-squares estimation (Tukey, 1983). Actually, the $L_{2}$ - norm estimator is the most familiar $M$-estimator. Another $M$ - estimator is the $L_{1}$-norm estimator and converges 
to the median estimator. The proposed estimator Var-norm can be viewed as a novel $M$-estimator, motivated by the need for assuring fidelity to the measured data in the SR image reconstruction task via a simple form estimator. Having studied estimators and their influence curves (Tukey, 1983), the $\psi$-function of a robust $M$ - estimator should have certain properties discussed below and presented in Table 1. In contradiction with the $L_{2}$ and $L_{1}$ estimators, the proposed estimator Var- norm presents all the desired properties.

TABLE 1 CRITERIA FOR $\psi$ REGARDING THE COMPARED ERROR NORMS.

\begin{tabular}{|c|c|c|c|}
\hline $\begin{array}{c}\text { Estimator/ } \\
\text { Criterion for } \psi\end{array}$ & $L_{2}-$ norm & $L_{1}-$ norm & Var-norm \\
\hline Resistant & No & Yes & Yes \\
\hline Bounded & No & Yes & Yes \\
\hline Moderate Continuity & Yes & No & Yes \\
\hline Finite Rejection Point & No & No & Yes \\
\hline Linear at Origin & Yes & No & Yes \\
\hline Odd & Yes & Yes & Yes \\
\hline
\end{tabular}

An estimator is resistant only if its breakdown bound (Tukey, 1983) is greater than zero. As a single observation is made larger, the mean increases without bound. Thus, the $L_{2}-$ norm estimator presents zero breakdown bound. However, the $L_{1}$ - norm estimator is resistant since its influence function retains constant values independently of the existence of contaminations in the observations. As far as the proposed estimator Var-norm is concerned, it is resistant as well. Its influence function presents variant slope, thus in the existence of outliers the Var- norm estimator is automatically altered to weaken their effect in its estimate. Regarding the resistance property, outlier frames stand for the contaminations. Furthermore, the influence function of an estimator is bounded if this estimator presents finite gross-error sensitivity (g.e.s.) (Tukey, 1983). The $L_{2}-$ norm estimator has infinite g.e.s. because its influence function increases linearly and without bound. Contrarily, the $L_{1}$ error norm presents finite g.e.s. due to its influence function retaining constant values. The proposed estimator Var- norm also presents finite gross error sensitivity. Explicitely, since the slope of its influence function varies according to the estimation error, the effect that a contaminated observation can have on the estimate is controlled. Concerning the moderate continuity property, the $L_{1}$ - norm estimator presents infinite 1.s.s (Tukey, 1983). However, the $L_{2}$-norm estimator as well as the proposed estimator Var- norm exhibit finite 1.s.s., since the supremum of the absolute slopes of chords which join all pairs of distinct points on the influence curves of both above-mentioned estimators is finite. Additionally, the rejection point (Tukey, 1983) of the $L_{2}$ and $L_{1}$ estimators is infinity because their influence functions do not reach zero. On the contrary, the proposed estimator Var-norm does exhibit finite rejection point. Since the slope of the $\psi_{\text {var }}$-function varies in relation to the estimation error, for very large error the slope becomes so small that the influence function tends to reach zero. Also, the $L_{2}-$ and Var-norm $\psi$-functions are both linear at origin. However, the $L_{1}$ influence function is not linear at origin. Finally, the $\psi_{L_{2}}-, \psi_{L_{1}}-$ and $\psi_{\text {var }}$ - functions are odd.

\section{Experiments}

\section{Simulated Experiments}

Two simulated experiments employing different scenes were conducted to compare the performance of the proposed SR technique with that of two existing SR techniques (Farsiu et al., 2004a, Patanavijit and Jitapunkul, 2006b) for SR reconstruction by the factor of 4 . At each experiment, a synthesized LR sequence that consists of 16 frames is employed. Fig. 3 depicts the experimental data of the "China air pollution" scene. The original China HR image is depicted in Fig. 3(a). Fig. 5 demonstrates the "Pollution over Mexico City" scene data. The original Mexico HR image is shown in Fig. 5(a). Both prementioned are satellite images provided by NASA (NASA). In the following, the procedure of creating a LR sequence from each one of the original HR images is described. First, the HR image is convolved with a symmetric Gaussian lowpass filter of size $4 \times 4$ and standard deviation equal to 1 , for simulating the effect of camera PSF. Then, the resulting image is down-sampled by the factor of 4 through pixel extraction. Thus, the first LR frame is created. As far as the rest frames are concerned, they are created via the pre-described procedure preceded by sub-pixel shifting the original HR image utilizing different motion vectors. Fig. 3(b) and 5(b) demonstrate a LR frame from the synthesized China and Mexico frame sequences, respectively. A subtle blend of Gaussian, speckle and Poisson noise is also inserted in the frames. Actually, Gaussian white noise of zero mean and variance 0.001 as well as speckle noise of variance 0.001 are employed. The corresponding noisy LR frames are depicted in Fig. 3(c) and 5(c). 


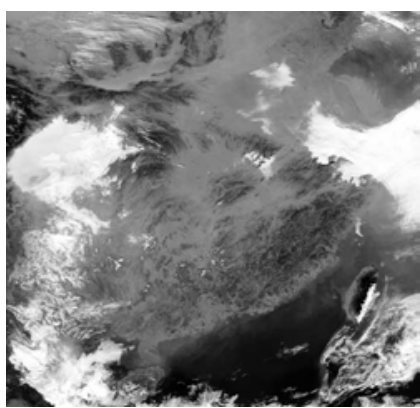

(a)

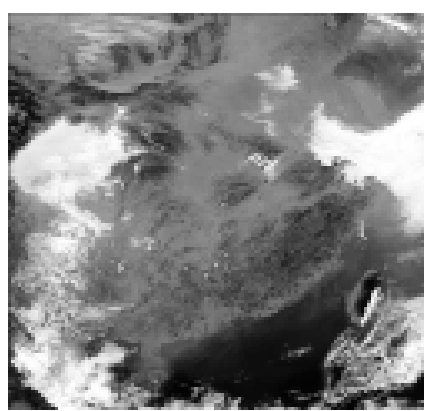

(b)

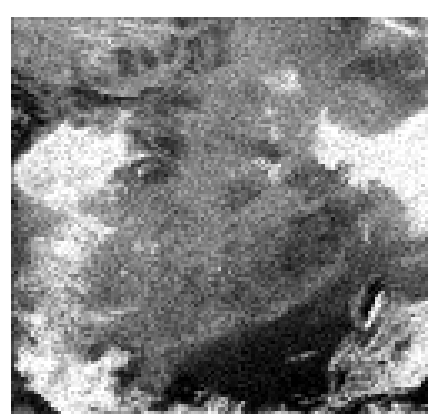

(c)

FIG. 3 "CHINA AIR POLLUTION" EXPERIMENTAL DATA. (a) THE ORIGINAL HR IMAGE (NASA). (b) A LR FRAME OF THE SYNTHESIZED SEQUENCE. (c) A LR FRAME CORRUPTED BY A SUBTLE BLEND OF GAUSSIAN, SPECKLE AND POISSON NOISE. REGARDING GAUSSIAN AND SPECKLE NOISE, VARIANCE EQUAL TO 0.001 IS EMPLOYED.

TABLE 2 THE PARAMETERS $R, \beta, a$ AND $\lambda$ UTILIZED BY THE TESTED METHODS FOR THE DIFFERENT SCENES EMPLOYED (CHINA/MEXICO/TEXT). THE VALUE OF $R$ IS RENEWED DURING EACH ITERATION AND IS RELATED WITH THE RECONSTRUCTION ITERATIONS NUMBER DENOTED BY $n$.

\begin{tabular}{|c|c|c|c|}
\hline Method/Parameter & Var - norm + BTV & $L_{1}-$ norm+BTV & $L_{2}-$ norm+BTV \\
\hline$R$ & $n / n / 10 n$ & --- & --- \\
\hline$\beta$ & $0.05 / 0.05 / 4$ & $0.05 / 0.025 / 2$ & $0.1 / 0.1 / 40$ \\
\hline$a$ & $0.3 / 0.3 / 0.2$ & $0.2 / 0.2 / 0.4$ & $0.3 / 0.4 / 0.1$ \\
\hline$\lambda$ & $0.3 / 0.32 / 0.4$ & $0.25 / 0.55 / 0.045$ & $0.05 / 0.04 / 0.2$ \\
\hline
\end{tabular}

The particular experiments are simulated experiments, but outliers are present. Data points exhibiting different distribution characteristics than those assumed by the considered image acquisition model are called outliers. Apart from outlier pixels that are present in every frame, outlier frames can also exist (Farsiu et al., 2004a). In fact, here outliers result from the original HR image shifting task. The motion vectors associated with each LR frame are known. However, an interpolation-based shifting procedure lacking accuracy is followed, which essentially introduces a bias. Additionally, outliers are caused by ignoring, in the image acquisition model which is formulated for performing the SR reconstruction task, the noise introduced in the LR frames. With regard to the initialization HR image of the SR reconstruction concerning all the tested methods, a median estimation procedure was applied at the noisy frames to obtain a "clean" frame which is then bi-linearly interpolated.

Table 2 presents the values of the stability factor $R$, the values of the gradient step size denoted $\beta$ as well as the values of the BTV term parameters $a$ (Panagiotopoulou and Anastassopoulos, 2009) and $\lambda$. Fig. 4 and 6 demonstrate parts of the SR reconstructed images. Regarding both scenes employed, visual comparison asserts predominance of the Var-norm + BTV method over the techniques in (Farsiu et al., 2004a) and (Patanavijit and Jitapunkul, 2006b), which employ the $L_{1}-$ and $L_{2}$ - norms, respectively, in combination with the bilateral TV regularizer. Thus, the proposed method performs resolution enhance-
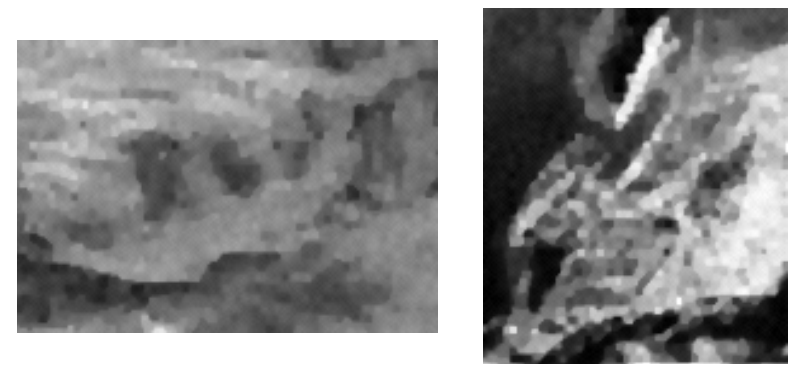

(a)
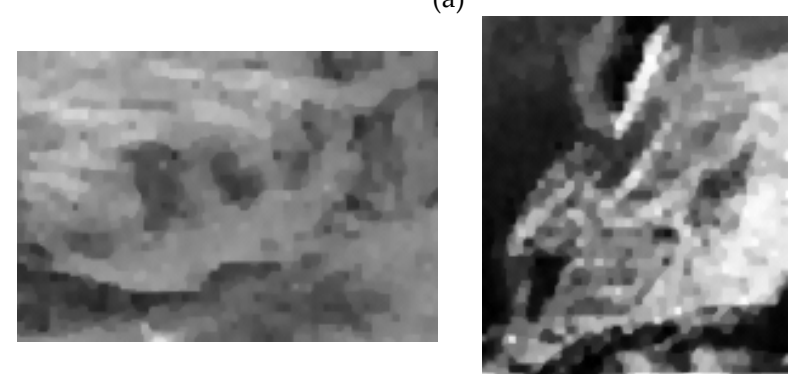

(b)
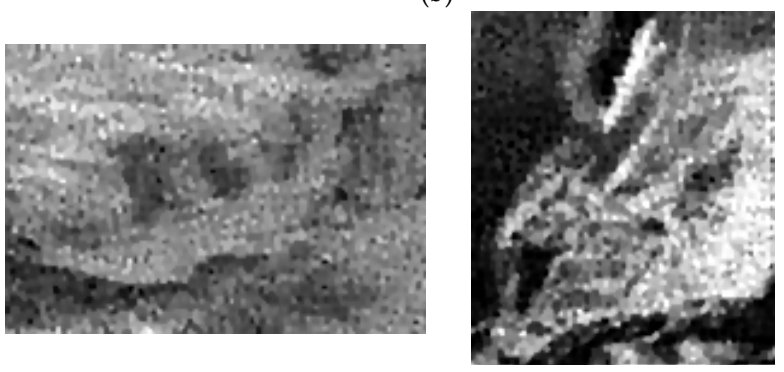

(c)

FIG. 4 PARTS OF THE SR RECONSTRUCTED IMAGES IN THE CASE OF THE CHINA SCENE (a) Var - NORM+BTV

TECHNIQUE (b) $L_{1}$ - NORM+BTV TECHNIQUE (Farsiu et al.,

2004a) (c) $L_{2}-$ NORM+BTV TECHNIQUE (Patanavijit and Jitapunkul, 2006b). THE PROPOSED METHOD EXHIBITS THE BEST PERFORMANCE. THE METHOD IN (b) OUTPERFORMS THE METHOD IN (c). 


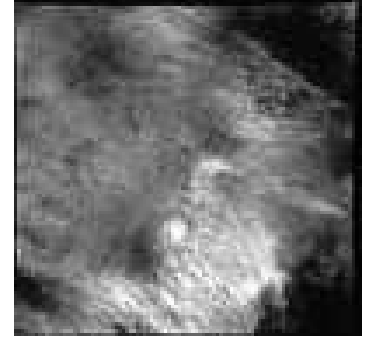

(a)

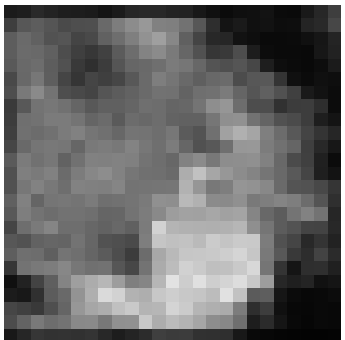

(b)

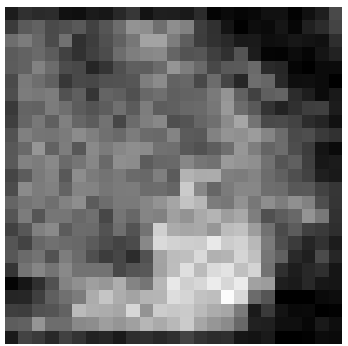

(c)

FIG. 5 "POLLUTION OVER MEXICO CITY" EXPERIMENTAL DATA. (a) THE ORIGINAL HR IMAGE (NASA). (b) A LR FRAME OF THE SYNTHESIZED SEQUENCE. (c) A LR FRAME CORRUPTED BY A SUBTLE BLEND OF GAUSSIAN, SPECKLE AND POISSON NOISE. REGARDING GAUSSIAN AND SPECKLE NOISE, VARIANCE EQUAL TO 0.001 IS EMPLOYED.
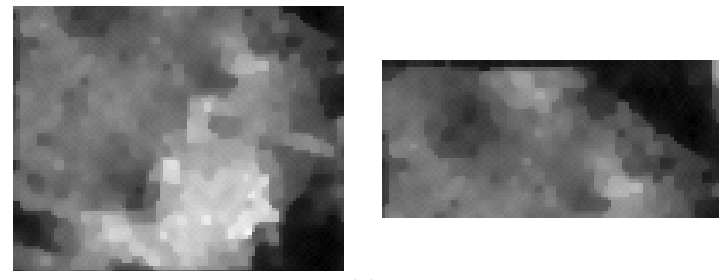

(a)
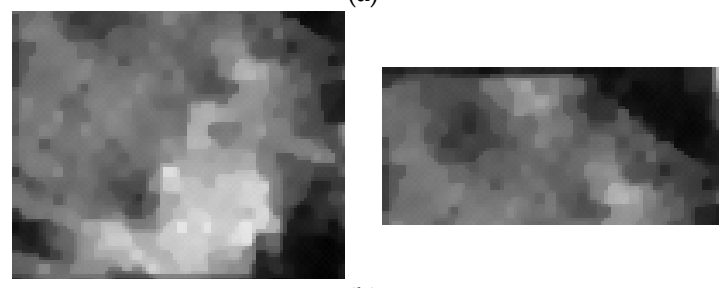

(b)
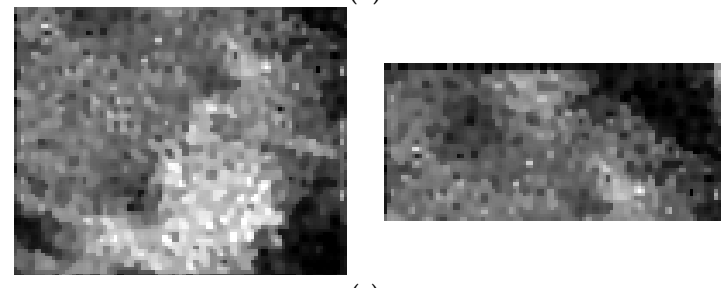

(c)

FIG. 6 PARTS OF THE SR RECONSTRUCTED IMAGES IN THE CASE OF THE MEXICO SCENE (a) Var - NORM+BTV

TECHNIQUE (b) $L_{1}-$ NORM+BTV TECHNIQUE (Farsiu et al.,

2004a) (c) $L_{2}-$ NORM+BTV TECHNIQUE (Patanavijit and

Jitapunkul, 2006b). THE PROPOSED METHOD OUTPERFORMS BOTH EXISTING TECHNIQUES. THE METHOD IN (b) OUTPERFORMS THE METHOD IN (c).

ment as well as noise removal. In contradiction, the technique in (Farsiu et al., 2004a) gives a smooth image with no edge preservation whilst noise is present at the image resulting from (Patanavijit and Jitapunkul, 2006b).

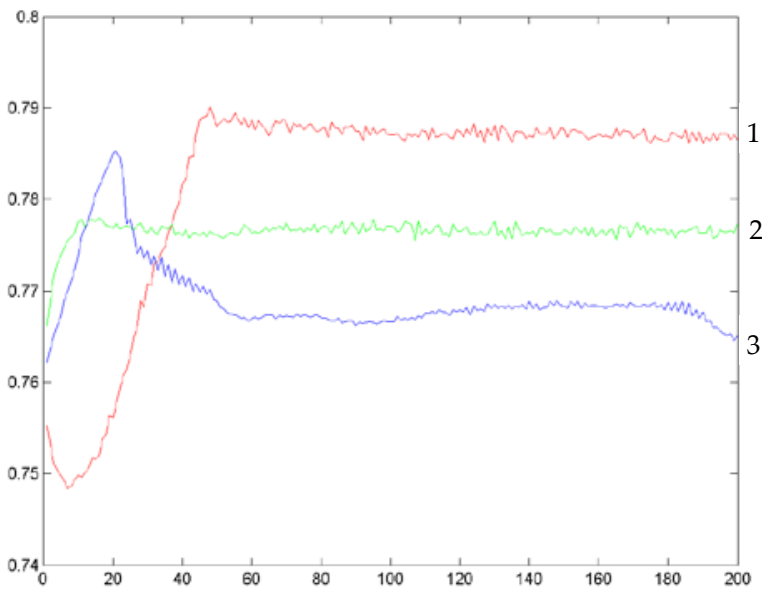

(a)

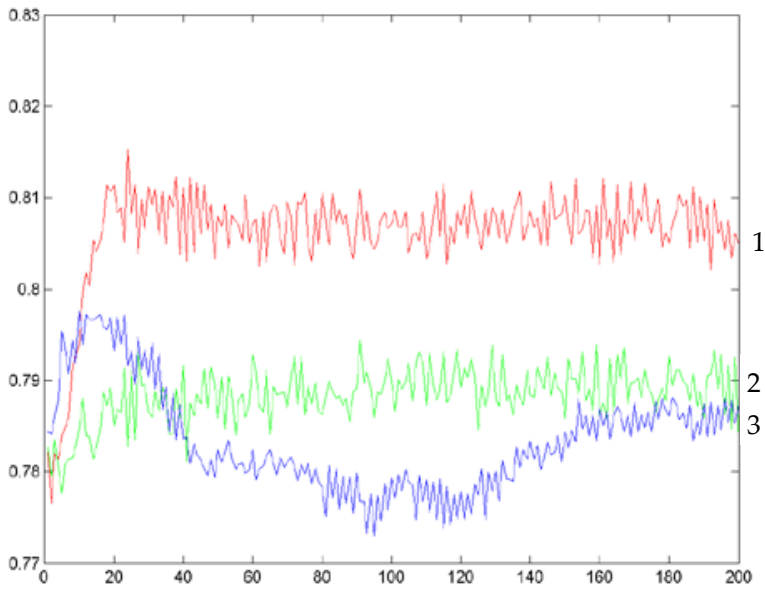

(b)

FIG. 7 PLOTS OF THE XYDEAS AND PETROVICH NUMERICAL MEASURE (Xydeas and Petrovich, 2000) VERSUS ITERATIONS NUMBER OF THE SR RECONSTRUCTION FOR THE TESTED SR METHODS (RED CURVE 1: Var - NORM + BTV, GREEN CURVE 2: $L_{1}-$ NORM + BTV (Farsiu et al., 2004a), BLUE CURVE 3:

$L_{2}-$ NORM + BTV (Patanavijit and Jitapunkul, 2006b)) IN THE CASE OF (a) CHINA SCENE (b) MEXICO SCENE. THE PROPOSED TECHNIQUE Var - NORM + BTV EXHIBITS THE BEST PERFORMANCE.

In Fig. 7 the numerical measure Xydeas and Petrovich (Xydeas and Petrovich, 2000) is plotted versus the number of iterations of the SR reconstruction procedure for the two employed scenes China and Mexico. The specific plots help to watch closely the performance of each technique. In both scene cases, the Var-norm + BTV method performs superior to the $L_{1}-$ and $L_{2}-$ norm + BTV techniques. Thus, in the specific diagrams, the curve of the proposed technique remains at higher values compared to the curves of the other two techniques. Actually, the $L_{1}-$ norm + BTV and $L_{2}-$ norm + BTV techniques come second and third, respectively, in SR performance. In addition, 
both plots demonstrate that early in iterations of the reconstruction procedure no comparison of the techniques can take place, because of the corresponding curves getting mixed. Thus, the direct comparison of the techniques can take place only when these curves clearly show the ranking in the methods performance. Actually, the measure Xydeas and Petrovich is in accordance with visual assessment regarding the performance of the tested SR methods. Better performance means larger Xydeas and Petrovich. The particular measure is an objective test of edge information between two images and takes values in the range $[0,1]$. Its value is 0 when the images under comparison do not share any edge information, whereas its value is 1 when there is no loss of edge information.

\section{Real Experiment}

In this work experimentation utilizing a real sequence of frames takes place as well, for SR image reconstruction by the factor of 4 . The employed real image sequence Text was drawn from Peyman Milanfar' s webpage (Farsiu et al., 2004a). This LR sequence consists of 30 frames that approximately follow the global translational motion model. With regard to the experimental procedure, the MDSP software (MDSP, 2004) is employed for the motion estimation task. Also, a $3 \times 3$ Gaussian kernel with standard deviation equal to 1 is assumed for the unknown PSF of the camera which captured the Text frame sequence. Regarding the initialization HR image of the SR reconstruction, a median estimation procedure was applied at the frames to obtain an improved frame which was then bi-linearly interpolated. Table 2 presents the parameter values that are employed at the real experiment.

Fig. 8(a) depicts a LR frame of the Text sequence. The SR reconstructed image resulting from the proposed technique is shown in Fig. 8(b). The corresponding results coming from the techniques in (Farsiu et al., 2004a) and (Patanavikit and Jitapunkul, 2006b) are demonstrated in Fig. 8(c) and (d), respectively. Visual comparison asserts that the Var-norm + BTV method performs superiorly to the techniques in (Farsiu et al., 2004a, Patanavijit and Jitapunkul, 2006b). Thus, in the image coming from the proposed technique the text letters have been successfully reconstructed and are legible. However, the other two SR techniques (Farsiu et al., 2004a, Patanavijit and Jitapunkul, 2006b) do not manage efficient reconstruction of the scene and artifacts are present. Specifically, the $L_{1}-$ norm + BTV technique comes second in SR performance and the $L_{2}-$ norm + BTV technique is third. Only visual comparison can be carried out here in the real SR reconstruction experiment since no original HR image is available.

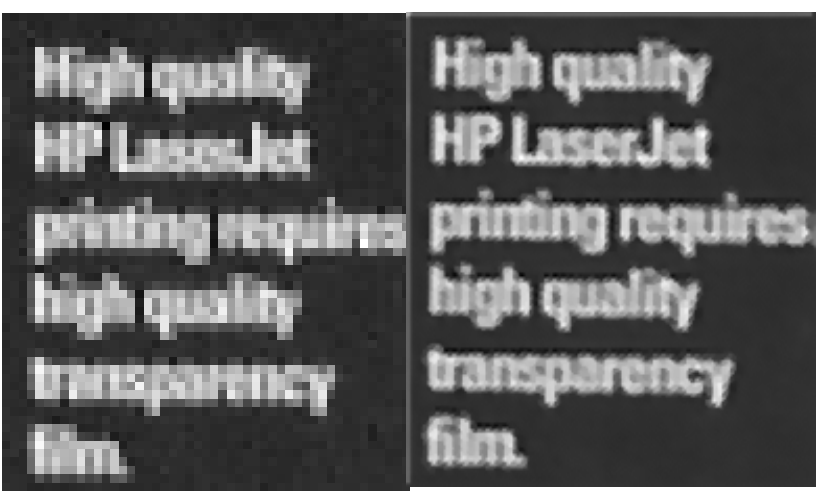

(a)

(b)

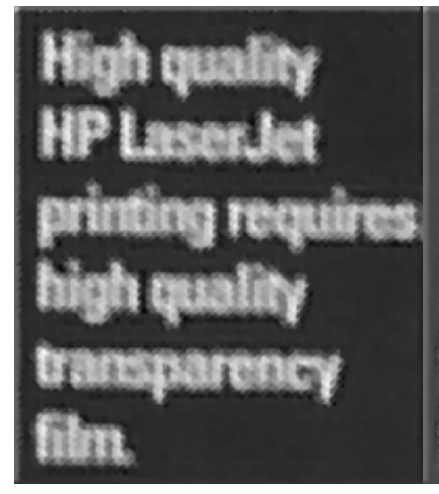

(c)

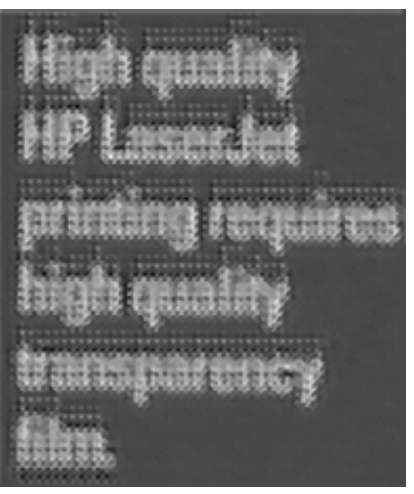

(d)
FIG. 8 THE SR RECONSTRUCTED IMAGES IN THE REAL TEXT EXPERIMENT. (a) LR FRAME. (b) THE SR IMAGE OBTAINED BY MEANS OF THE PROPOSED METHOD. (c) THE SR IMAGE RESULTING FROM THE TECHNIQUE IN (Farsiu et al., 2004a). (d) THE SR IMAGE CREATED BY MEANS OF THE METHOD IN

(Patanavijit and Jitapunkul, 2006b). THE PROPOSED TECHNIQUE PERFORMS BEST. THE TECHNIQUES IN (c) AND (d) ARE SECOND AND THIRD, RESPECTIVELY, IN PERFORMANCE.

\section{Discussion}

In the present work, the direct comparison of the Var- , $L_{1}-$ and $L_{2}-$ norm estimators takes place. Thus, their influence functions are scaled so as to return values in the same range (Black et al., 1998). With regard to the parameter values selection, experimentation proved that there are more than one values of the gradient step size $\beta$ for which a method converges to the desired answer. In fact, for each method there has an upper value of $\beta$ over which the search for the global minimum is of no success. Over the entire range of appropriate $\beta$ values, the value that gives a reconstruction procedure both fast and exhibits less oscillatory movements was chosen (Panagiotopoulou and Anastassopoulos, 2009). 

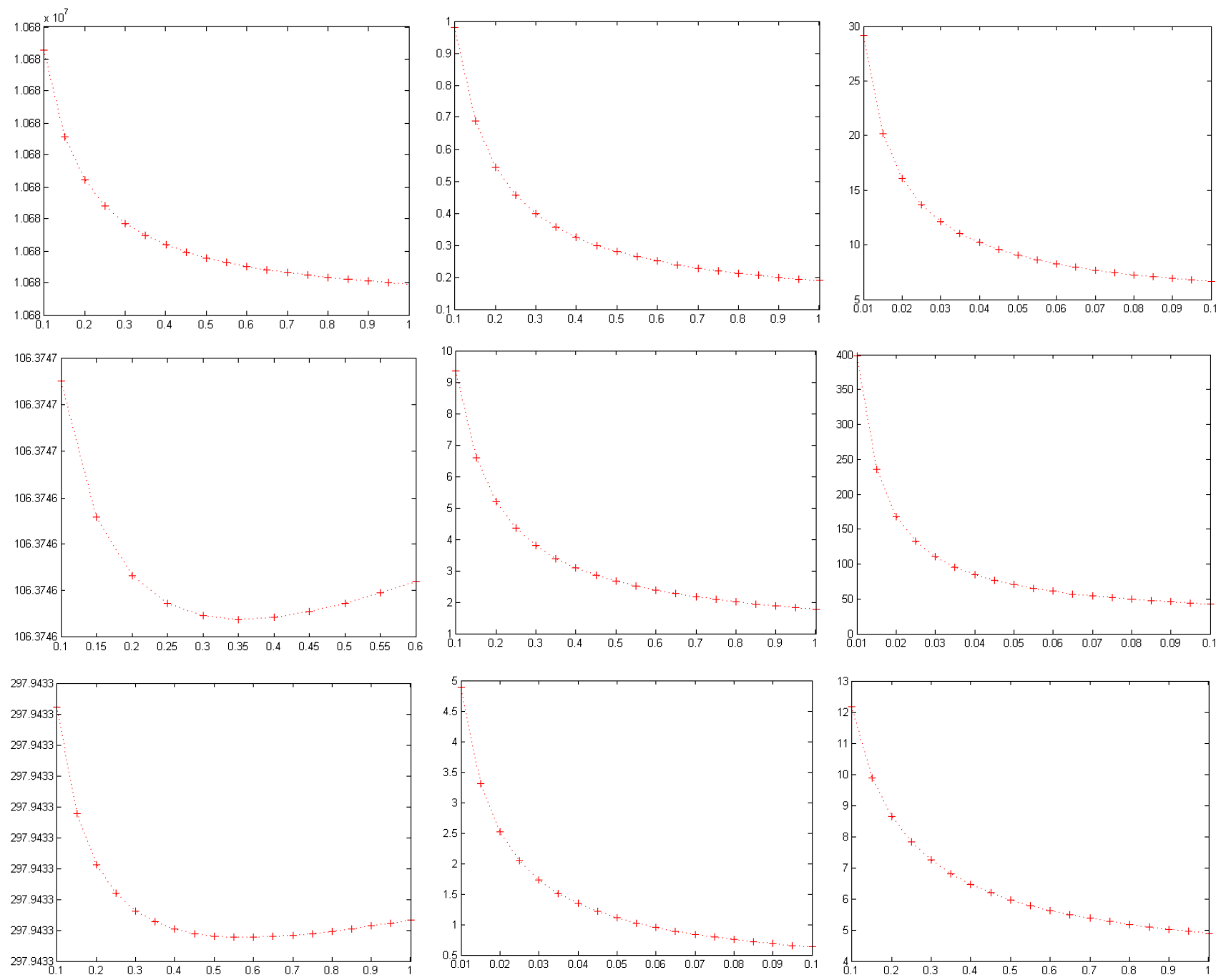

FIG. 9 U-CURVE (Yuan et al., 2010) PLOTTED VERSUS THE REGULARIZATION PARAMETER $\lambda$ FOR THE THREE TESTED METHODS AND SCENES. THE PLOTS REGARDING THE PROPOSED TECHNIQUE ARE SHOWN AT THE FIRST COLUMN WHILST THE SECOND AND THIRD COLUMNS CORRESPOND TO THE TECHNIQUES IN (Farsiu et al., 2004a) AND (Patanavijit and Jitapunkul, 2006b). THE FIRST, SECOND AND THIRD ROWS DEPICT THE RESULTS FOR THE CHINA, MEXICO AND TEXT SCENES, RESPECTIVELY. THE LEFT MAXIMUM CURVATURE POINT OF THE CURVE IS REGARDED AS THE OPTIMAL PARAMETER.

As far as the regularization parameters $a$ and $\lambda$ are concerned, their selection is interdependent. First, the appropriate value for the parameter $a$ is set manually by visual inspection. Afterwards, the optimal value of the parameter $\lambda$ is selected by means of the U-curve technique presented in (Yuan et al., 2010). Thus, a Ucurve function has been constructed using the datafidelity term and the regularization term. The left and right sides of this curve correspond to the regularization parameter $\lambda$ for which the data-fidelity term and the prior term are dominated by each other. Actually, the more horizontal part corresponds to the parameter for which the data-fidelity and the prior terms are close to each other, and this part is where the optimal parameter exists. The maximum curvature point close to the left vertical part of the curve is regarded as the optimal regularization parameter. Fig. 9 depicts the plots of the U-curve function versus the regularization parameter $\lambda$ for the three tested methods and the scenes employed China, Mexico and Text. Selecting the values of both parameters $a$ and $\lambda$ by means of the U-curve technique is a future research goal.

\section{Conclusions}

In this work, a new stochastic regularized SR image reconstruction method was presented. Actually, a novel estimator named Var - norm was proposed for employment in the data-fidelity term of the multiframe SR problem formulation. The introduced estimator was based on the variance of the SR estimation error which can be elicited from the difference between the estimated LR frame and the corresponding measured LR frame. In fact, the proposed influence function allowed during the SR 
reconstruction procedure the automatic renewing of the characterization of the bias introduced in the solution by each measurement. The novel estimator Var-norm in combination with the BTV regularizer was proposed to perform the task of SR image reconstruction. The experimental results prove that the introduced technique outperforms the existing SR techniques which employ the $L_{1}-$ and $L_{2}$ - norm estimators in combination with the BTV regularization. Thereafter, the Var-norm estimator predominates over the $L_{1}-$ and $L_{2}$-norm estimators. Actually, the Var-norm estimator presents an adaptive nature and adjusts the influence given to each measurement according to the associated estimation error. However, the $L_{1}$ - norm estimator gives all measurements a constant weight of one whilst the $L_{2}$-norm estimator gives to each measurement a weight which is twice the measurement value.

\section{ACKNOWLEDGMENT}

The author would like to thank Prof. V. Anastassopoulos for his valuable comments that helped to improve the clarity of the presentation of the proposed estimator.

\section{REFERENCES}

Baker S., and Kanade T. "Limits on super-resolution and how to break them." IEEE PAMI 24 (2002): 1167-1183.

Black M. J., et al. "Robust Anisotropic Diffusion." IEEE Transactions on Image Processing 7 (1998): 421-432.

El-Yamany N. A., Papamichalis P. E., and Schucany W. R. "A robust image super-resolution scheme based on redescending M-estimators and information-theoretic divergence." ICASSP 2007 (2007): I-741 to I-744.

El-Yamany N. A., Papamichalis P. E., and Christensen M. P. "Adaptive framework for robust high-resolution image reconstruction in multiplexed computational imaging architectures." Applied Optics 47 (2008): B117-B127.

El-Yamany N. A., and Papamichalis P. E. "Using boundedinfluence M-estimators in multi-frame super-resolution reconstruction: A comparative study." ICIP (2008): 337340.

Engl H. W., Hanke M., and Neubauer A. Regularization of Inverse Problems, Kluwer Academic Publishers, Netherlands, 2000.

Farsiu S. et al. "Fast and Robust Multiframe Super-
Resolution." IEEE Transactions on Image Processing 13 (2004): 1327-1344.

Farsiu S., et al. "Advances and Challenges in SuperResolution." International Journal of Imaging Systems and Technology 14 (2004): 47-57.

Ivanovski Z. A., Panovski L., and Karam L. J. “Robust superresolution based on pixel-level selectivity." SPIE Proceedings on Visual Communications and Image Processing 20066077 (2006): 607707-1 to 607707-8.

Katsaggelos A. K., Molina R., and Mateos J. Super Resolution of Images and Video, Synthesis Lectures on Image, Video and Multimedia Processing, Morgan and Claypool Publishers, USA, 2007.

MDSP resolution enhancement software, 2004.

NASA Image Exchange (NASA Technical Reports Center)

Panagiotopoulou A., and Anastassopoulos V. "Superresolution image reconstruction employing Kriging interpolation technique." Proceedings of IWSSIP 2007 \& EC-SIPMCS 2007, Maribor, Slovenia, (2007): 151-154.

Panagiotopoulou A., and Anastassopoulos V. "Scanned images resolution improvement using neural networks." Neural Computing and Applications 17 (2008): 39-47.

Panagiotopoulou A., and Anastassopoulos V. "Superresolution image reconstruction techniques: Trade-offs between the data-fidelity and regularization terms." Information Fusion 13 (2012): 185-195.

Panagiotopoulou A., and Anastassopoulos V. "SuperResolution Reconstruction of Thermal Infrared Images." Proceedings of the $4^{\text {th }}$ WSEAS International Conference on Remote Sensing, Venice, Italy, (2008): 40-44.

Panagiotopoulou A., and Anastassopoulos V. "Regularized super-resolution image reconstruction employing robust error norms." Optical Engineering 48 (2009): 117004-1 to 117004-14.

Panagiotopoulou A. "Multi-frame super-resolution image reconstruction employing the novel estimator L1invnorm." IWSSIP 2012, Vienna, Austria, (2012): 562-565.

Park S. C., Park M. K., and Kang M. G. “Super-resolution image reconstruction: A technical overview." IEEE Signal Processing Magazine 20 (2003): 21-36.

Patanavijit V., and Jitapunkul S. "An Iterative SuperResolution Reconstruction of Image Sequences using a Bayesian Approach with BTV prior and Affine BlockBased Registration." Proceedings of $3^{\text {rd }}$ Canadian 
Conference on Computer and Robot Vision 00 (2006): 45.

Patanavijit V., and Jitapunkul S. "An iterative superresolution reconstruction of image sequences using a Bayesian approach and affine block-based registration." EUSIPCO, 2006.

Patanavijit V., and Jitapunkul S. "An Iterative SuperResolution Reconstruction of Image Sequences using Affine Block-Based Registration." Proceedings of the 2006 International Conference on Wireless Communications and Mobile Computing (2006): 51-56.

Patanavijit V., and Jitapunkul S. "A Robust Iterative Multiframe Super-Resolution Reconstruction using a Huber-Bayesian Approach with Huber-Tikhonov Regularization." ISPACS (2006): 13-16.

Patanavijit V., Sermwuthisarn P., and Jitapunkul S. "A robust iterative super-resolution reconstruction of image sequences using a Tukey's biweigth Bayesian approach with fast affine block-based registration." IEEE International Conference on Multimedia and Expo (2007): 480-483.

Patanavijit V., and Jitapunkul S. "A Lorentzian Stochastic Estimation for a Robust Iterative Multiframe SuperResolution Reconstruction with Lorentzian-Tikhonov Regularization." EURASIP Journal on Advances in Signal Processing (2007): 1-21.

Pham T. Q., Vliet L. J., and Schutte K. “Influence of signal-tonoise ratio and point spread function on limits of superresolution." SPIE Proceedings on Image Processing: Algorithms and Systems IV 5672 (2005): 169-180.

Pham T. Q., Vliet L. J., and Scutte K. "Robust super- resolution by minimizing a Gaussian-weighted $L_{2}$ error norm." Journal of Physics: Conference Series 124, $4^{\text {th }}$ AIP International Conference and the $1^{\text {st }}$ Congress of the IPIA (2008): 1-8.

Pickup L. C., Roberts S. J. and Zisserman A. "A Sampled Texture Prior for Image Super-Resolution." Advances in Neural Information Processing Systems Conference, 2004. Tsagaris V., Panagiotopoulou A., and Anastassopoulos V. "Interpolation in multispectral data using neural networks." Proceedings of SPIE 5573 (2004): 460-470.

Tukey J. Understanding Robust and Exploratory Data Analysis, J. Wiley and Sons, 1983.

Xydeas C. S., and Petrovic V. "Objective image fusion performance measure." Electronics Letters 36 (2000): 308309.

Yuan Q., Zhang L., Shen H., and Li P. "Adaptive MultipleFrame Image Super-Resolution Based on U-curve." IEEE Transactions on Image Processing 19 (2010): 3157-3170.

Antigoni Panagiotopoulou was born in Greece. She received a B.Sc. in Physics in 2002, a M.Sc. degree in Electrical Engineering in 2004 and her Ph.D. in Electrical Engineering in 2009, all from the University of Patras, Greece. Now, she is a researcher in the Chemical Engineering Department, University of Patras and the Institute of Chemical Engineering Sciences of the Foundation for Research and Technology-Hellas, Patras, Greece.

Her research interests include: a) image resolution enhancement including interpolation and super-resolution reconstruction techniques, neural networks and scanner function modeling b) evaluation of atmospheric chemical transport models using satellite data c) atmospheric particle morphology and microscopy studies. 